\title{
The Arabidopsis ATK1 gene is required for spindle morphogenesis in male
}

\section{meiosis}

\author{
Changbin Chen ${ }^{1,2, *}$, Adam Marcus ${ }^{1}$, Wuxing $\mathrm{Li}^{1,2,3}$, Yi Hu${ }^{1,2}$, Jean-Philippe Vielle Calzada4,5, \\ Ueli Grossniklaus ${ }^{4,6}$, Richard J. Cyr ${ }^{1,3}$ and Hong Ma1,2,3,† \\ 1Department of Biology, The Pennsylvania State University, University Park, PA 16802 USA \\ 2The Life Sciences Consortium, The Pennsylvania State University, University Park, PA 16802 USA \\ 3The Intercollege Graduate Program in Plant Physiology, The Pennsylvania State University, University Park, PA 16802 USA \\ ${ }^{4}$ Cold Spring Harbor Laboratory, Cold Spring Harbor, NY 11724 USA \\ ${ }^{5}$ CINVESTAV-Irapuato, Plant Biotechnology Unit, CP 36500, Irapuato, GTO, Mexico \\ ${ }^{6}$ Institute of Plant Biology, University of Zürich, $\mathrm{CH}-8008$, Zürich, Switzerland \\ *Present address: University of Pennsylvania, Plant Science Institute, Biology Department, Philadelphia, PA 19104, USA \\ †'Author for correspondence (e-mail: hxm16@psu.edu) \\ Accepted 25 February 2002
}

\section{SUMMARY}

The spindle plays a central role in chromosome segregation during mitosis and meiosis. In particular, various kinesins are thought to play crucial roles in spindle structure and function in both mitosis and meiosis of fungi and animals. A group of putative kinesins has been previously identified in Arabidopsis, called ATK1-ATK4 (previously known as KATA-KATD), but their in vivo functions have not been tested with genetic studies. We report here the isolation and characterization of a mutant, atk1-1, which has a defective ATK1 gene. The atk1-1 mutant was identified in a collection of $D s$ transposon insertion lines by its reduced fertility. Reciprocal crosses between the atk1-1 mutant and wild type showed that only male fertility was reduced, not female fertility. Molecular analyses, including revertant studies, indicated that the $D s$ insertion in the $A T K 1$ gene was responsible for the fertility defect. Light microscopy revealed that, in the atk1-1 mutant, male meiosis was defective, producing an abnormal number of microspores of variable sizes. Further cytological studies indicated that meiotic chromosome segregation and spindle organization were both abnormal in the mutant. Specifically, the atk1-1 mutant male meiotic cells had spindles that were broad, unfocused and multi-axial at the poles at metaphase I, unlike the typical fusiform bipolar spindle found in the wild-type metaphase I cells. Therefore, the $A T K 1$ gene plays a crucial role in spindle morphogenesis in male Arabidopsis meiosis.

Key words: Arabidopsis thaliana, Kinesin, Meiosis, Spindle, Transposon

\section{INTRODUCTION}

The microtubule-based spindle plays an essential role in chromosome segregation during mitosis and meiosis (Hoyt and Geiser, 1996; Dawe, 1998). At prometaphase and metaphase, microtubules extending from the spindle poles and attaching to the chromosomes at the kinetochore (kinetochore microtubules) and on the arms are responsible for chromosome congression at the equator (Hoyt and Geiser, 1996). After the separation of homologs at meiosis I, or of sister chromatids at meiosis II and mitosis, movement of chromosomes to the poles depends on the kinetochore microtubules. The elongation of the spindle with extension of non-kinetochore microtubules further distances the two groups of chromosomes. Therefore, microtubule-based movement is central to chromosome segregation.

Kinesins are microtubule-based motor proteins (Howard, 1996; Mandelkow and Hoenger, 1999; Sharp et al., 2000). The first kinesin was discovered in squid axoplasm and shown to be involved in organelle translocation (Brady, 1985; Vale et al., 1985). Since then, a large family of related proteins (for brevity, they are referred to as 'kinesins' hereafter) (Bloom and Endow, 1994) has been isolated from a wide range of organisms (Scholey et al., 1985; Saxton et al., 1988; McCaffrey and Vale, 1989; Roof et al., 1991; O'Conell et al., 1993; Liu and Lee, 2001). The members of the kinesin superfamily share a conserved region of about 340 amino acids within the putative motor domain (Goodson et al., 1994). Kinesins typically generate a motive force along a microtubule by hydrolyzing ATP, similar to dynein, the first described microtubule motor (Milisav, 1998). The first described kinesin (often referred to as conventional kinesin) and its close relatives move toward the 'plus' end of microtubules and contain an N-terminal motor domain with ATP and microtubule-binding sites, an $\alpha$-helical coiled-coil central stalk region, and a C-terminal globular tail that binds to cargo (Scholey et al., 1989; Yang et al., 1989; Yang et al., 1990). Other kinesins contain a motor domain near the $\mathrm{C}$ terminus and 
typically move towards to the minus end of microtubules, whereas some members with an internal motor domain may affect microtubule stability. Non-motor flanking region(s) of kinesins are highly diverged and are thought to have a variety of functions, including organelle translocation, vesicle deposition, mitosis and cytokinesis.

Several kinesins have been found to be associated with mitosis and/or meiosis (Endow, 1999b; Hildebrandt and Hoyt, 2000; Mountain and Compton, 2000). For example, the fission yeast Cut7 protein is localized to mitotic and meiotic spindles (Hagan and Yanagida, 1992). Furthermore, in budding yeast, kinesins have also been found to be important for structure and positioning of the mitotic spindle (Hoyt et al., 1992; Saunders and Hoyt, 1992; Cottingham and Hoyt, 1997). In Drosophila, the Non-Claret Disjunctional (NCD) protein is associated with both mitotic and meiotic spindles and involved in their assembly or structural integrity (Hatsumi and Endow, 1992; Endow et al., 1994; Matthies et al., 1996; Sharp et al., 2000). Another Drosophila kinesin, No Distributive Disjunction (NOD), has also been found to regulate meiotic spindle assembly (Theurkauf and Hawley, 1992). The studies in fungi and animals have revealed multiple roles for kinesins during mitosis, including spindle assembly, spindle positioning and chromosome movement.

Molecular genetic analysis has identified several Arabidopsis members of the kinesin superfamily (Liu and Lee, 2001; Reddy and Day, 2001). One of these is the kinesin-like calmodulin-binding protein (KCBP) (Reddy et al., 1996), encoded by the ZWICHEL gene required for normal branching during trichome development (Oppenheimer et al., 1997). Several other Arabidopsis kinesin genes were identified using PCR with degenerate primers and are called KAT genes (KATAKATD) (Mitsui et al., 1993; Mitsui et al., 1994; Tamura et al., 1999; Liu and Lee, 2001). To conform to Arabidopsis nomenclature guidelines and to avoid confusion with the KAT1 gene encoding a potassium channel (Schachtman et al., 1994), we have renamed these genes ATK1-ATK4, for Arabidopsis thaliana kinesins. The ATK1, ATK2 and ATK3 proteins are similar in sequence to yeast KAR3 and Drosophila NCD proteins, and contain a motor domain near the $\mathrm{C}$ terminus, an $\alpha$-helical coiled coil region and a smaller globular $\mathrm{N}$-terminal domain. Among these, the ATK2 and ATK3 proteins are very similar to each other $(83 \%$ amino acid identity over the entire length). In addition, the ATK1 protein is also quite similar to both ATK2 and ATK3 (57\% and 56\% amino acid sequence identity, respectively). The ATK1 (KATA) protein is thought to be involved in the functioning of the spindle apparatus, owing to its localization within the midzone of the mitotic spindle (Liu et al., 1996). In addition, it has been suggested that ATK1 is a minus-end motor (Liu et al., 1996), consistent with its sequence similarity with other C-terminal kinesins known to be minus-end motors (Song et al., 1997; Endow, 1999a); this has recently been confirmed in vitro using recombinantly expressed ATK1 protein (Marcus et al., 2002).

However, the in vivo functions of the ATK genes are not clear because of the lack of mutants and relevant functional studies using genetic approaches. In particular, it is not known whether any of these genes are involved in meiotic spindle functions and chromosome segregation. We now report the isolation of a new Arabidopsis mutant with greatly reduced male fertility, and demonstrate that the mutation is in the ATK1 gene. We have designated the mutant allele as atk $1-1$ and show that the atk1-1 mutant is defective in meiotic spindle morphogenesis and chromosome segregation, providing direct evidence that the ATKl gene is important for spindle function in male meiosis.

\section{MATERIALS AND METHODS}

\section{Plant material and growth conditions}

The Arabidopsis thaliana wild-type and mutant plants used in this study were of the Landsberg erecta (Ler) ecotype. The atkl-1 mutant (initially designated at ET4786) carries a Ds element and was isolated from a collection of $D s$ insertion lines generated as described previously (Sundaresan et al., 1995). All plants were grown in the greenhouse under 18 hours light and 6 hours darkness, at $20-24^{\circ} \mathrm{C}$. For reversion analysis, the atkl-1 mutant was crossed with a line (HM108) carrying the stabilized Ac element Ac2 (Sundaresan et al., 1995), and nine mutant plants that produced revertant sectors of normal fertility were identified in the $\mathrm{F}_{2}$ generation.

\section{Molecular analysis of the mutant locus}

Southern hybridization experiments (not shown) detected a single Ds element in the atk1-1 mutant. To obtain Arabidopsis genomic sequences flanking this Ds element, DNA from four individual atk1-1 plants was isolated and used as templates for TAIL PCR experiments (thermal asymmetric interlaced PCR) (Liu et al., 1995). Ds-specific primers from both the 5' and 3' ends of Ds, Ds5-1, Ds5-2, Ds5-3, Ds31, Ds-3-2 and Ds3-3, were used in combination with AD degenerate primers (Liu et al., 1995; Grossniklaus et al., 1998). A PCR fragment of approximately $0.4 \mathrm{~kb}$ was obtained using Ds3-1/Ds-3-2 with AD2, and yielded approximately $330 \mathrm{bp}$ sequence information. A search of the GenBank database revealed that the sequence adjacent to the 3' end of the Ds element matched that of the ATK1 gene. Using the Arabidopsis genomic sequence, we designed two primers: oMC413, 5'-TCCACTTTCCTTCGCTTGTCAAA-3'; and oMC414, 5'-ATTGTCGGTAACCCGAATTTAGG-3'. These primers, as well as Dsspecific primers, were used to analyze wild-type, mutant and revertant DNAs.

\section{Isolation and subcloning of the ATK1 CDNA}

The ATK1 cDNA was isolated from a cDNA library that was generously provided by June Nasrallah's laboratory, using primers synthesized based on published ATK1 sequence (Mitsui et al., 1993): forward\#1, 5'-GTCGACATGGCTTCTCGCAACCAG-3'; and reverse\#1, 5'-TGCCATAGCTTAAGCGAGAG TCAACG-3'. The PCR product was cloned into the Invitrogen TA cloning pCR 2.1TOPO vector, yielding plasmid pAM01, and the insert was confirmed by sequence analysis. Two subclones were generated by cloning PCR fragments into the invitrogen pCR II TOPO vector for the purpose of synthesizing in situ hybridization probes. The first subclone (designated pAM02) was generated with the full-length cDNA clone as the template and the following primers: forward\#2, 5'-GTGGACATGGCTTCTCGCAACCAG-3'; and reverse\#2, 5'-AAGCTTGTGTTCTC-3'. The second subclone (designated pAM03) was generated similarly using primers: forward\#3, 5' -TCACTAGTGAGCTCATGGCTTCTCGCAAC-3'; and reverse\#3, 5'-TCGGATCCGGCGCGCCTGCAAGCTTTTCATGTAG-3'.

\section{In situ RNA hybridization}

Sections of wild-type and atk1-1 mutant inflorescences were prepared and hybridized with labeled probes essentially as described previously (Drews et al., 1991; Flanagan and Ma, 1994). To synthesize the antisense probe, the pAM02 plasmid was digested with XhoI and used for in vitro transcription with the T7 polymerase. For the control sense probe, the pAM02 plasmid was digested with BamHI and transcribed 
with the SP6 polymerase. The hybridization signal was detected using a photosensitive emulsion and visualized using a Nikon microscope (Nikon, Melville, NY) and an Optronics digital camera (Optronics, Goleta, CA).

\section{Light and scanning electron microscopy}

Inflorescences with immature floral buds of up to $\sim 2 \mathrm{~mm}$ in length were collected and placed in Carnoy's fixative (ethanol:chloroform:glacial acetic acid=6:3:1). The floral buds were fixed at room temperature for at least 4 hours before being stored in a freezer at $-20^{\circ} \mathrm{C}$. Immunofluorescence microscopy was performed as described (Brown and Lemmon, 1995). Samples were examined using a Nikon microscope and photographed with an Optronics digital camera. The images were further processed using a Photoshop 5.5 (Adobe Systems, San Jose, CA).

Chromosome spreads and DAPI staining were performed according to Ross et al. (Ross et al., 1996). The fixed tissue (in Carnoy's fixative) was washed twice with water and twice with $10 \mathrm{mM}$ citrate buffer $\mathrm{pH}$ 4.5. They were digested with $0.3 \%$ cytohelicase, $0.3 \%$ cellulase and $0.3 \%$ pectolyase in citrate buffer for 30 minutes at $37^{\circ} \mathrm{C}$. They were then washed with citrate buffer and stored at $4^{\circ} \mathrm{C}$. Before observation, a single digested floral bud was dissected from the inflorescence and placed in a small drop of $60 \%$ acetic acid on a slide. The anthers were dissected and moved away from cell debris, and the slide was tapped to release microspore mother cells (MMCs). DAPI (4,6-diamino-2phenylindole dihydrochloride) solution (Vectorshield from Vector Laboratories, Burlingame, CA) $(5 \mu \mathrm{l})$ was added onto the slide, which was then covered with a cover glass. Immunofluorescence microscopy was performed with a monoclonal anti- $\beta$-tubulin antibody (Chemicon International, Temecula, CA) as previously described (Peirson et al., 1996). The chromosome spread and immunofluorescence samples were examined using a Nikon microscope and images were digitally recorded. Pollen grains for scanning electron microscopy were prepared as described before (Chen et al., 2000). Samples were examined by using a JEOL (JSM) 5400 SEM.

\section{RESULTS}

\section{Isolation and initial characterization of a male fertility mutant}

To identify genes important for male reproduction, we screened for mutants that displayed reduced fertility among Arabidopsis insertion lines carrying modified versions of the maize $D s$ element. One of the lines (ET4786) had reduced fertility; unlike the wild type with fully grown siliques, the mutant produced many siliques of reduced size (Fig. 1A), particularly from early arising flowers (not shown). Only approximately

Fig. 1. Wild-type, atk1-1 and revertant phenotypes. (A) A region of an atk1-1 plant with both mutant branch (arrow) and $A c$-induced revertant sectors (arrowhead) that had normal siliques. (B) A wildtype flower. (C) An atk1-1 flower. (D) A wild-type tetrad with four microspores. (E) A product of atkl-1 meiosis, showing six microspores of unequal size. (F) Wild-type pollen grains of uniform size are brightly stained, indicating that they are functional. (G) atkl1 pollen grains of variable sizes; most were faintly stained, indicating that they are dead. $(\mathrm{H})$ Polar view of a wild-type pollen grain, with three apertures (arrows) that partition the pollen surface equally. (I) A side view of a wild-type pollen grain, showing the long apertures. (J) An atk1-1 pollen grain, showing that three apertures (arrows) were located unevenly on the pollen surface. (K) An atkl-1 pollen grain, showing that the apertures (arrows) were shorter than those of the wild-type pollen. Scale bars: $0.5 \mathrm{~cm}$ in A; $0.5 \mathrm{~mm}$ in B,C; $25 \mu \mathrm{m}$ in $\mathrm{D}-\mathrm{G} ; 10 \mu \mathrm{m}$ in $\mathrm{H}-\mathrm{K}$.
$15 \%$ of mutant siliques were of the normal size; even such normal-sized siliques often had a reduced number of seeds (not shown), resulting in about $10 \%$ fecundity when compared with the wild type. This fertility defect was male specific, as shown by reciprocal crosses with the wild type. Because we learned subsequently that the Ds element was inserted into the ATK1 gene, we named this mutant atk1-1. Because the $D s$ element carries a KanR marker, the mutant was also kanamycin resistant. When the mutant was crossed to the wild-type, the $F_{1}$ progeny were normal, indicating that the mutation was recessive. In addition, among $\mathrm{F}_{2}$ plants from such a cross, about three quarters were kanamycin resistant and one third of the Kanamycin-resistant plants were mutant, indicating that the mutation was linked to the $D s$ element. Selfed progeny from the mutant plants were all kanamycin resistant and had reduced male fertility.

To determine the cause of reduced male fertility in the atk1-1 mutant, we compared wild-type and mutant pollen development. Similar to the wild-type flower (Fig. 1B), the atk1-1 mature flower produced normal floral organs, but had reduced number of pollen grains (Fig. 1C and data not shown). Furthermore, in the atkl-1 mutant, meiosis produced a variable number of microspores. In the wild type, all observed tetrads had four spores each (Fig. 1D; $n=200$ ); by contrast, the number of microspores per meiosis in the atk $1-1$ mutant ranged from three to ten with the following percentages $(n=200): 0.5 \%$ (three spores), $3.5 \%$ (four spores), $11.5 \%$ (five spores), $15 \%$ (six spores), $43.5 \%$ (seven spores), $16 \%$ (eight spores), $8.5 \%$ (nine spores) and $1.5 \%$ (ten spores) (e.g. Fig. 1E). Whereas all wild-type pollen grains are functional (Fig. 1F), only a small fraction of atkl-1 pollen grains developed to maturity and were viable (Fig. 1G).

Moreover, the atkl-1 mature pollen had an abnormal pollen wall surface. Scanning electron microscopy of a wild-type pollen grain (Fig. $1 \mathrm{H})$ shows the three apertures that partition the surface equally. By contrast, in the atkl-1 mutant, only $17 \%$

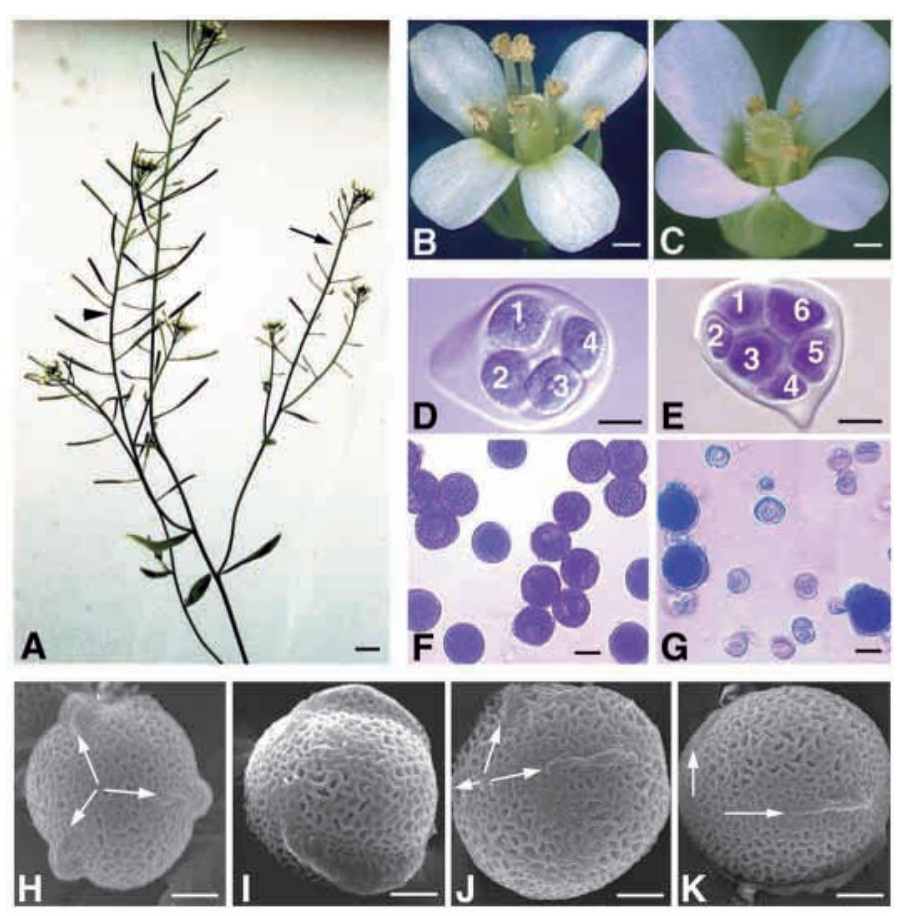


of pollen grains had three apertures; furthermore, the apertures did not always divide the pollen surface into three equal parts (Fig. 1J). In addition, the apertures on the mutant pollen (Fig. $1 \mathrm{~K}$ ) were shorter than those on the wild-type pollen (Fig. 1I). The other $83 \%$ of pollen grains had only one or two aperture(s) on the pollen surface (data not shown); these pollen grains were much smaller than normal, similar to the non-viable ones.

\section{The fertility defect is due to a $D s$ insertion in the ATK1 gene}

As mentioned above, the atkl-1 mutant carries a cosegregating $D s$ insertion. If this $D s$ insertion caused the mutant phenotype, the excision of the $D s$ should produce revertant sectors with a normal phenotype. To generate revertant sectors, we crossed the atk1-1 mutant with an Ac-carrying line; out of $102 \mathrm{~F}_{2}$ plants, nine mutant plants produced 21 fertile branches that were fully fertile (Fig. 1A), in contrast to the mutant, which had only infrequent normal-sized siliques. These normal branches produced flowers with normal pollen grains (not shown), unlike the abnormal pollen grains of the mutant, ruling out the possibility that the seeds of the revertant sector were due to contaminating pollen. Furthermore, when seeds of more than ten revertant sectors were planted, both normal and mutant plants were observed for each revertant, indicating that the sectors were ATK1/atk1-1 heterozygous, consistent with the sectors being revertants. Therefore, the atkl-1 mutant was caused by a $D s$ insertion.

To identify the disrupted gene in the atkl-1 mutant, we determined $\sim 330$ bp sequences adjacent to the $3^{\prime}$ end of the $D s$ element from a $0.4 \mathrm{~kb}$ fragment obtained using the TAIL-PCR procedure (Liu et al., 1995). Database searches revealed that this fragment matched the genomic sequence of the ATK1 gene, and that the $D s$ element was inserted into the first intron (Fig. 2A; 53 bp downstream of the $3^{\prime}$ end of the first exon). Using primers (oMC413 and oMC414) that match genomic sequences flanking the $D s$ element, we amplified a PCR fragment of the expected size from wild-type and revertant genomic DNAs, but not from mutant DNAs (data not shown). We then determined the sequences of four revertants around the $D s$ insertion site, and found that one had the wild-type sequence and three had 6 or $7 \mathrm{bp}$ insertions (Fig. 2B), as expected for $D s$ excision footprints. Because these small insertions are in the first intron, they are not expected to affect the $A T K 1$ gene function. These results demonstrate that the $D s$ insertion in the ATK1 gene was responsible for the defects in male meiosis and fertility.

\section{The ATK1 gene is expressed in male meiotic cells}

Originally, the ATK1(KATA) cDNA was cloned from an Arabidopsis cDNA library made from leaf and floral mRNAs and later shown to be expressed in seedlings by immunoblot analysis (Mitsui et al., 1993; Liu et al., 1996). To learn more about its spatial distribution in the flower, particularly in the anther, we performed RNA in situ hybridization experiments using a gene-specific probe of a region of the ATK1 cDNA, including the $5^{\prime}$ non-translated region and the coding region for the $\mathrm{N}$ terminus of ATK1. As shown in Fig. 3, ATK1 is expressed in the flower at several stages, including
A.

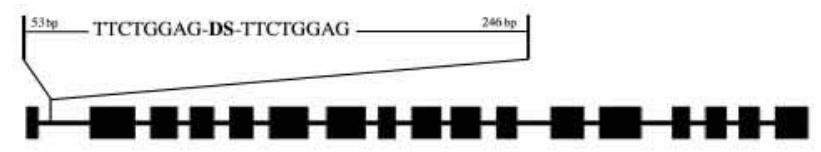

Fig. 2. Analysis of the ATK1 locus. (A) A diagram of the ATK1 gene with exons shown as boxes and introns as lines. The $D s$ element is inserted in the first intron, with the $8 \mathrm{bp}$ duplication $53 \mathrm{bp}$ downstream of the $3^{\prime}$ end of the first exon and 246 bp upstream of the $5^{\prime}$ end of the second exon. (B) Sequence near the Ds insertion site in the wild type (WT), atk $1-1$ and three revertants (rev1, rev5 and rev6); the inserted nucleotides in atkl-1 and the revertants are underlined.

stamen and pistil primordia (not shown), immature petal and anther (Fig. 3A), male meiotic cells (Fig. 3B) and ovules (data not shown). As a control, atk 1-1 mutant floral sections showed no specific hybridization signal (Fig. 3C and data not shown); this is consistent with the fact that the $D s$ element contains transcriptional termination sequences and truncated mRNAs are usually unstable. Therefore, ATK1 is preferentially expressed in tissues containing dividing cells, including male meiocytes.

\section{atk1-1 is defective in homologous chromosome segregation in male meiosis}

To investigate the mutant defect further, we analyzed wild-type
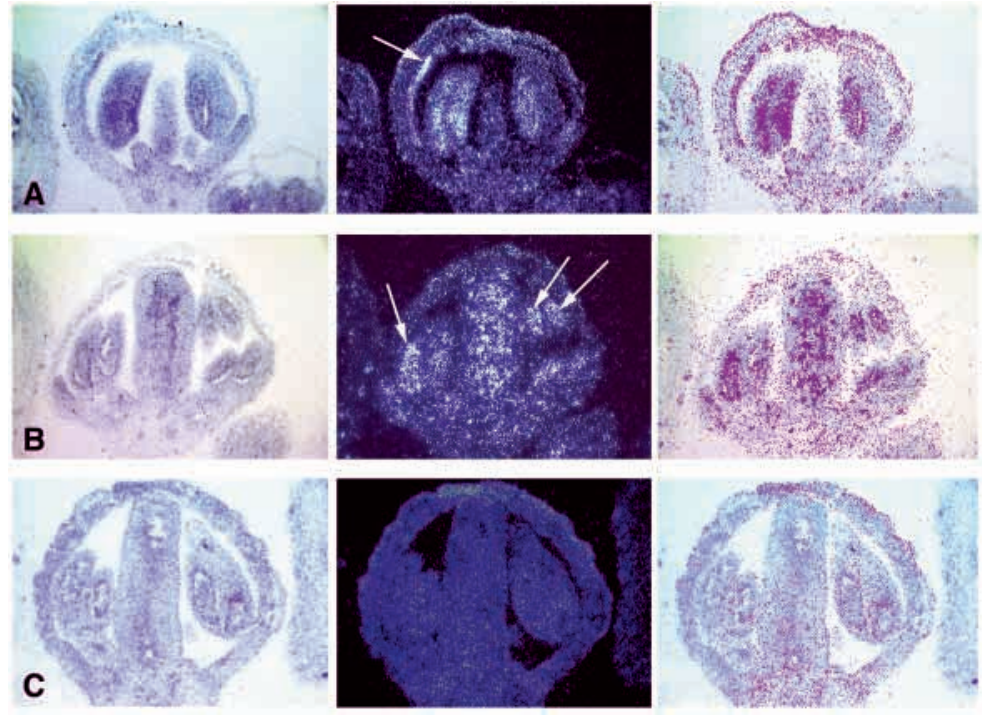

Fig. 3. In situ RNA hybridization. For each row of three panels, the left panel is a bright-field image, the center one is a dark-field image and the right one a composite using Photoshop. (A,B) Wild-type floral sections hybridized with the ATK1 antisense RNA. (A) A stage 8 floral bud with ATK1 transcripts in young anthers before meiosis and the tip of a petal primordium (arrow). (B) A stage 9 floral bud with male meiocytes (arrows) in the anther and the developing pistil, both showing ATK1 expression. (C) An atk1-1 section of a stage 9 flower, showing background levels of silver grains. 
and mutant meiosis in early arising flowers using a chromosome spread and DAPI staining procedure (Ross et al., 1996) (Fig. 4). At leptotene (not shown), chromosomes begin to condense and form barely visible thin lines. Homologous chromosomes begin pairing at zygotene (Fig. 4A) and complete synapsis at pachytene, seen as thick lines (Fig. 4B). Chromosomes further condense at diplotene (Fig. 4C), and five highly condensed bivalents are visible at diakinesis (Fig. 4D). We observed that the atkl1 mutant appeared normal during male meiotic prophase I, from leptotene (not shown) through zygotene and pachytene (Fig. 4E,F), to diplotene and diakinesis (Fig. 4G,H).

In the wild-type male meiocyte at metaphase I (Fig. 4I), the five bivalents congress and align in parallel at the equatorial plane. Homologues separate at anaphase I (Fig. 4J) and form two groups of partially decondensed chromosomes at telophase I (Fig. 4L). By contrast, the congression of bivalents was incomplete in the atkl-1 mutant, with some chromosome(s) failing to localize completely at the equator (arrow in Fig. 4M); furthermore, chromosomes failed to align in parallel (arrowheads in Fig. 4M,K,O). Homolog separation at anaphase I was also abnormal (Fig. 4K,N,O). Sometimes, one pair of homologues would separate first (arrow in Fig. 4N); other times, one pair would remain attached after the other four had already separated (arrows in Fig. 4K,O). Therefore, atkl-1 male meiotic chromosomes were clearly abnormal in alignment at metaphase I and in segregation at anaphase I, and were probably also affected in congression during prometaphase I. Nevertheless, the distribution of chromosomes at anaphase I suggests that homologous chromosomes could, at least sometimes, distribute to either side of the equator in atkl-1 cells (not shown), providing an explanation for the observed infrequent viable pollen and partial fertility. At telophase I, although two groups of chromosomes could be seen in the mutant cells, the chromosomes were somewhat scattered (Fig. 4P), perhaps owing to an abnormal spindle structure.

During meiosis II in the wild-type cells, the two groups of non-homologous chromosomes are clearly separated by a zone of organelles, indicative of meiosis II (Fig. 4Q); the chromosomes are fully condensed again at metaphase II (Fig. 4R). Then, sister chromatids separate at anaphase II (Fig. 4S), and decondense at telophase II (Fig. 4T). In the atkl-1 mutant, the distribution of non-homologous chromosomes was often abnormal, which is probably due to the defective chromosome segregation in meiosis I. In addition, the organelles did not formed the characteristic narrow zone; instead they were more diffuse (Fig. 4U,V,W). Chromosomes condensed at prophase II (Fig. 4U), and became fully condensed at metaphase II (Fig. 4V). The sister chromatids separated at anaphase II to form 20 chromosomes (Fig. 4W), which formed multiple small clusters of decondensing chromosomes at
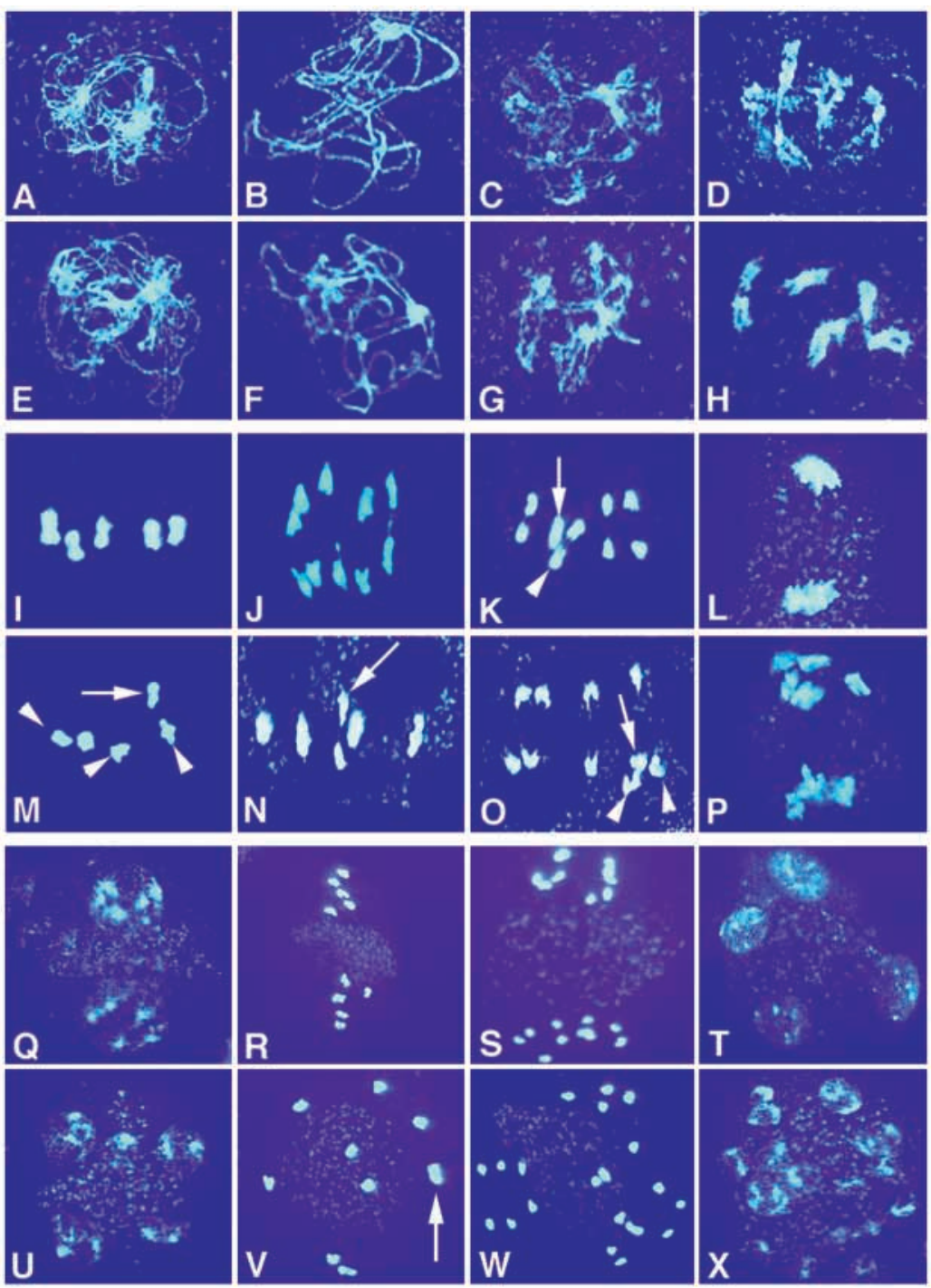

Fig. 4. Wild-type (A-D,I,J,L,Q-T) and atkl-1 (E-H,K,M-P,U-X) male meiosis from chromosome spreads. (A,E) Zygotene, with condensed chromosomes seen as thin threads. (B,F) Pachytene, showing synapsis of homologous chromosomes, forming thick threads. $(\mathrm{C}, \mathrm{G})$ Diplotene with partial separation of homologs. (D,H) Diakinesis with condensed bivalents of attached homologs. (I) Metaphase I, with five bivalents at the equator. (J) Anaphase I, showing separated homologs. (L) Telophase I, with two groups of chromosomes. (M) Metaphase I of atk1-1, with one bivalent (arrow) located slightly away from the equator; three others (arrowheads) are not aligned in parallel with each other. (N) Metaphase Ianaphase I transition in the atkl-1 mutant, showing one pair of separated homologs and four bivalents. (K,O) Anaphase I in atk1-1 meiocytes, showing four pairs separated homologs and one remaining bivalent (arrows); at least one pair of homologs are misaligned (arrowheads). (P) Telophase I in atk1-1.

(Q,U) Prophase II. (R,V) Metaphase II. (S,W) Anaphase II. (T,X) Telophase II. In the wild type, a narrow zone of organelles is present between the separated homologs (Q-S); however, the organelles were more diffuse in atkl-1 cells $(\mathrm{U}-\mathrm{W})$. Arrow in V indicates two superimposed chromosomes.

telophase II (Fig. 4X), resulting in the formation of many more than four nuclei. The number of chromosomes in groups varied in the atk1-1 mutant (not shown). As a consequence, spore number and size differed between individual meiotic events.

Although the atk1-1 mutant had normal female fertility, as indicated by pollination with normal pollen, it is possible that 
female meiosis in the atk1-1 mutant might have mild defects. We therefore examined female meiosis in wild-type and atkl-1 flowers. Our observations indicate that chromosome alignment at metaphase I and homolog segregation at anaphase I in female meiosis appeared normal in atkl-1 mutant (not shown). Therefore, the atkl-1 mutation did not seem to affect female meiosis.

\section{ATK1 is required for normal spindle formation in male meiosis}

The ATK1 gene encodes a putative kinesin (Mitsui et al., 1993; Marcus et al., 2002) and the atkl-1 mutant is defective in chromosome movement and segregation. Both predict that microtubule structures, particularly the meiotic spindle, would be affected in the atkl-1 mutant. To test this hypothesis, we performed immunofluorescence microscopy with an anti- $\beta$-tubulin antibody on early-arising flowers. At mid-prophase I, wild-type and atk1-1 microtubule distribution was perinuclear (Fig. 5A,E). At metaphase I, the wild-type spindle showed a typical bipolar and highly fusiform configuration, with chromosomes at the equator (Fig. 5B). However, the atkl-1 mutant spindle at metaphase I was unfocused at the two poles (Fig. 5F, $100 \%, n=258$ ), indicating a defect in spindle formation, particularly at the poles. In addition, the mass of microtubules seemed less than normal and there was a higher background of tubulin staining in the atkl-1 mutant. At the conclusion of anaphase I,
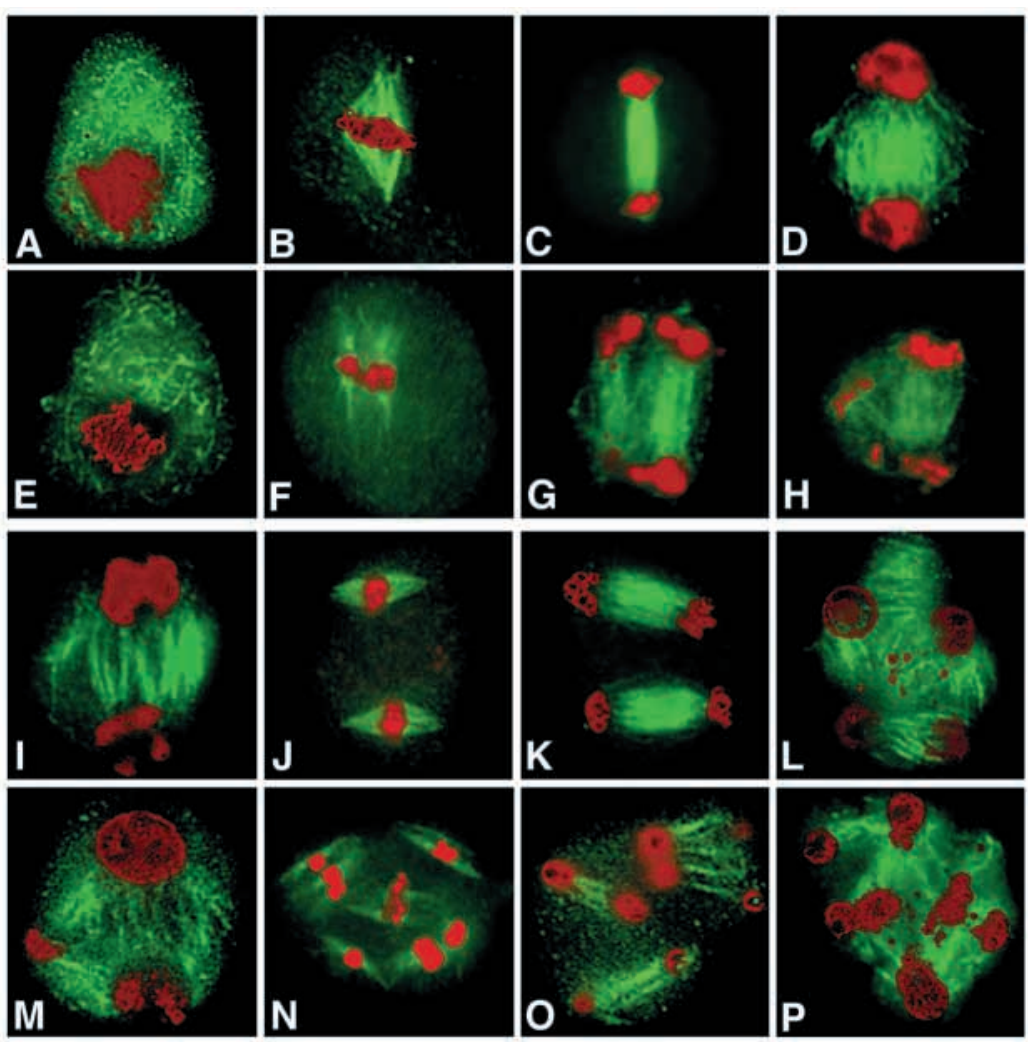

Fig. 5. Spindle structures in wild-type and atk1-1 male meiocytes. The microtubules are in green and chromosomes are in red. (A-D) Wild-type meiosis I. (E-H) atk1-1 meiosis I. (I-L) Wild-type meiosis II. (M-P) atk1-1 meiosis II. the interzonal microtubules in wild-type cells appear in a tight configuration between the recently separated chromosomes (Fig. 5C). However, at the same stage, the cells of the atk1-1 mutant displayed a broad and more diffuse collection of interzonal microtubules between an equally broad and disorganized distribution of recently separated chromosomes (Fig. 5G). At telophase I, a broad microtubule structure resembling the phragmoplast was formed between the two groups of chromosomes (Fig. 5D), but microtubules in the atk1-1 mutant were somewhat more disorganized and appeared to organize between the abnormally distributed chromosomes (Fig. 5H).

During wild-type meiosis II, microtubules between the two groups of chromosomes disappear (Fig. 5I) and two bipolar metaphase II spindles are formed (Fig. 5J). The spindles then become tightly cylindrical at anaphase II (Fig. 5K). New microtubule structures then form at telophase II in preparation for cytokinesis (Fig. 5L). In the atk1-1 mutant, although microtubules were found between clusters of chromosomes at prophase II (Fig. 5M), the metaphase II spindles were abnormal, with multiple mini-spindles, each organized around a small group of chromosomes (Fig. 5N). At anaphase II, spindles in the atkl-1 cells had an apparent reduction in the number of microtubules (Fig. 5O). Although the number of chromosomal clusters in the atk $1-1$ mutant was abnormal at telophase I, microtubules formed the expected structures between chromosomal clusters (Fig. 5P). These observations during meiosis I and II indicate that the atk1-1 mutant is specifically defective in the formation of metaphase and anaphase spindles, but did not exhibit an obvious defect in microtubule structures at prophase, nor telophase defects that were independent of the abnormal chromosome distribution.

\section{DISCUSSION}

We have isolated a new Arabidopsis mutant, atk1-1, in the ATK1 (KATA) gene encoding a kinesin. We observed that the atk1-1 mutant had reduced male fertility, reduced number of viable pollen grains, and abnormal male meiosis. Cytological analysis indicates that male meiosis in the atkl-1 mutant produced an abnormal number of microspores of variable size; this is likely to be a consequence of abnormal segregation of chromosomes at meiosis I. Immunolocalization studies further revealed that the meiotic spindle in the atk1-1 lacked the bipolar structure of the normal spindle. This abnormal spindle is the likely cause for the abnormal chromosome segregation and the subsequent spore and pollen defects. In addition, the failure of atkl-1 chromosomes to align properly at metaphase I can also be explained by the abnormal spindles.

The $D s$ insertion is in the first intron of the ATK1 gene. Because the $D s$ element contains transcriptional termination sequences, the transcription of $A T K 1$ is probably terminated upstream of the second exon, as supported by our RNA in situ hybridization results. Therefore, the atkl-1 insertion is probably an RNA null mutation. Our finding that the atkl-1 male metaphase I and II spindles are always abnormal supports the hypothesis that ATK1 is essential for normal male meiotic spindle assembly. The fact that in atkl-1 cells chromosome 
congression at metaphase I is close to complete and chromosome separation at anaphase I is often non-random may explain why a low percentage of pollen is still viable and the mutant is partially male fertile.

Because ATK1 is also expressed during seedling development (Liu et al., 1996) and in other cells of the flower (this paper), including cells in pistil and ovules, it may have functions other than controlling spindle formation in male meiosis. Previously, the ATK1 protein has been shown to be localized to the mid-zone of the mitotic spindle and to the phragmoplast (Liu et al., 1996), suggesting that it may have a function in these mitotic structures. The fact that atkl-1 is female fertile and has normal chromosome segregation in female meiosis is probably due to gene redundancy. In addition to the known ATK1 and ATK2-ATK4 genes, the Arabidopsis genome contains another gene (Accession Number, AL161503) encoding a kinesin that is $83 \%$ identical $(91 \%$ similar) to ATK1 over the entire length of the predicted protein (Arabidopsis Genome Initiative, 2000). We propose to name this gene ATK5; ATK5 probably fulfills the required ATK1 functions in female meiosis and mitosis.

Kinesins are motor proteins that typically move along microtubules. The ATK1 protein is a member of the C-terminal kinesin family that also includes the Drosophila NCD and the budding yeast KAR3 proteins. NCD is known to associate with the meiotic spindle and is required for normal spindle assembly (Hatsumi and Endow, 1992; Endow et al., 1994; Matthies et al., 1996). In meiotic cells of the ncd mutant, spindles are multipolar, diffuse or broad. The multipolar spindles are associated with bivalents that do not congress tightly. We have shown here that atk1-1 spindles are also broad, like some $n c d$ spindles. Therefore, atk1-1 meiotic spindles exhibit similarity to $n c d$ meiotic spindles. In mitosis, NCD was thought to bind one microtubule via its tail domain and move along another with its motor domain, thereby crosslinking microtubules (Karabay and Walker, 1999b; Karabay and Walker, 1999a; Sharp et al., 2000). It is possible that NCD can also crosslink microtubules during meiosis, thereby promoting the assembly of the bipolar spindle.

Mutants defective in other kinesins with C-terminal motors also exhibit abnormal spindle assembly (Bascom-Slack and Dawson, 1997; Prigozhina et al., 2001). For example, in yeast kar3 mutant cells, meiotic spindles are abnormally long and monopolar instead of bipolar, indicating a defect in spindle assembly. The kar3 meiotic cells also exhibit defects in chromosomal synapsis during prophase I and reduced meiotic recombination frequency, raising the possibility that KAR3 may play a role in chromosome interaction and recombination (Bascom-Slack and Dawson, 1997). In Aspergillus, the klpA1 mutation causes a transient inhibition of mitotic bipolar spindle formation; this spindle defect is enhanced by a mutation in the gene encoding a gamma-tubulin (Prigozhina et al., 2001).

The ATK1 protein might play a similar role to the other previously characterized C-terminal motors. Recombinantly expressed full-length ATK1 supports minus-end-directed microtubule motility in a non-processive manner (i.e. it likely detaches from the microtubule after each power stroke) (Marcus et al., 2002). The lack of processivity is consistent with its role in organizing microtubules (i.e. microtubules provide tracks for movement and ATK1 conveys other microtubules as cargo). These activities are consistent with a role for ATK1 in focusing meiotic spindle poles. The failure of the atk1-1 mutant to form bipolar male meiotic spindles strongly supports the idea that ATK1 is needed to organize microtubules at the two poles.

Microtubule-microtubule sliding is thought to be the basis of force generation in the spindle (Sharp et al., 2000). Experiments in budding yeast and Aspergillus show that mutations in the plus-end-directed motors, cin8/kipl and $\operatorname{bim} C$ can be suppressed by subsequent mutations in the minus-end-directed motors, $\operatorname{kar} 3$ and $k l p A$, respectively. In addition, the Drosophila NCD protein and bipolar kinesin KLP61F (BIMC homolog) also display antagonistic motor forces in the mitotic spindle (Sharp et al., 1999). Thus, plusend and minus-end-directed motors are thought to generate counteracting forces (i.e. plus-end-directed motors are producing outward-acting forces, while minus-end motors produce inward-directed forces) within the spindle to maintain spindle structure. The ATK1 protein shares $35 \%$ identity to KLPA and $33 \%$ identity to KAR3, suggesting that ATK1 might play a similar role in plant meiosis, by providing an inwardly directed force necessary for bipolar spindle assembly and maintenance. It is also worth noting that the amount of tubulin staining in the atk $1-1$ mutant spindles was considerably lower than that of the wild-type spindles. This suggests that the number of microtubules in the mutant spindles might be reduced.

Whereas the defect of atkl-1 meiotic cells in spindle formation is quite clear, other meiotic microtubule structures at prophase I and II, and telophase I and II seem quite normal. Therefore, ATK1 may be required only in spindle assembly, and not in the organization of other structures, such as the phragmoplast-like broad microtubule structure formed at telophase I. The phragmoplast is a plant-specific microtubule structure formed near the end of mitosis and facilitates cytokinesis by helping to deliver membrane vesicles that are needed for the formation of the cell plate. Furthermore, the Arabidopsis kinesin AtPAKRP1 was found to be associated with the mitotic phragmoplast (Lee and Liu, 2000). In some plants, meiosis I is followed by cytokinesis, producing two cells. In Arabidopsis, no cytokinesis occurs between meiosis I and II; nevertheless, a phragmoplast-like structure and an associated zone of membranous organelles are formed. Because atk1-1 mutant meiotic cells can still form these structures, other kinesins, such as AtPAKRP1, are probably involved in the assembly of this microtubule structure. In addition, previous studies of other C-terminal kinesins have not examined meiosis II spindles. We show here that atk1-1 metaphase II and anaphase II cells also have abnormal spindles that are diffuse and broad. Therefore, ATK1 is also required for spindle assembly during meiosis II in Arabidopsis.

Our studies have shown that ATK1 is important for plant meiotic spindle assembly. Although ATK1 is similar in sequence to NCD, KAR3 and KLPA, and may have some functional resemblance to these proteins, ATK1 may also have some functional differences compared with these other kinesins, as supported by the difference in details of the mutant phenotypes. The isolation of this mutant provides a new means of studying kinesin function in plant meiosis. Future analyses will provide insights into the mechanism of ATK1 action in plants. 
We thank James M. Moore and Wendy B. Gagliano for helping to generate Ds insertion lines; Alice Coluccio for her help in the sterility screen, Ruth Chou, Heather Barsh and Lisa Blickley for technical assistance; Ming Yang, Dazhong Zhao and Xiaohui Yang for helpful discussions; Rosemary Walsh for assistance with scanning electron microscopy; Anthony Omeis for plant care; and Bo Liu and John F. Kennedy for comments on this manuscript. This work was supported by funds from the Biology Department and Life Sciences Consortium to H. M., a grant from the US National Science Foundation to H. M., a US Department of Agriculture grant to H. M. and R. C., a US Department of Energy grant to R. C., and funds from the Cold Spring Harbor Laboratory President's Council and the Janggen-Poehn Stiftung to U. G. J.-P. V. C. was a recipient of a post-doctoral fellowship from the Fonds National Suisse de la Recherche Scientifique.

\section{REFERENCES}

Arabidopsis Genome Initiative (2000). Analysis of the genome sequence of the flowering plant Arabidopsis thaliana. Nature 408, 796-815.

Bascom-Slack, C. A. and Dawson, D. S. (1997). The yeast motor protein, Kar3p, is essential for meiosis I. J. Cell Biol. 139, 459-467.

Bloom, G. S. and Endow, S. A. (1994). Kinesin related proteins. Protein Profile 1, 1089-1105.

Brady, S. (1985). A novel brain ATPase with properties expected for the fast axonal transport motor. Nature 317, 73-75.

Brown, R. C. and Lemmon, B. E. (1995). Methods in plant immunolight microscopy. In Methods in Cell Biology: Methods in Plant Cell Biology, Part A. Vol. 49 (ed. D. W. Galbraith, D. P. Bourque and H. J. Bohnert), pp. 85107. New York: Academic Press.

Chen, C., Wang, S. and Huang, H. (2000). LEUNIG has multiple functions in gynoecium development in Arabidopsis. Genesis 26, 42-54.

Cottingham, F. R. and Hoyt, M. A. (1997). Mitotic spindle positioning in Saccharomyces cerevisiae is accomplished by antagonistically acting microtubule motor proteins. J. Cell Biol. 138, 1041-1053.

Dawe, R. K. (1998). Meiotic chromosome organization and segregation in plants. Annu. Rev. Plant Physiol. Plant Mol. Biol. 49, 371-395.

Drews, G. N., Bowman, J. L. and Meyerowitz, E. M. (1991). Negative regulation of the Arabidopsis homeotic gene AGAMOUS by the APETALA2 product. Cell 65, 991-1002.

Endow, S. A. (1999a). Determinants of molecular motor directionality. Nat. Cell Biol. 1, E163-E167.

Endow, S. A. (1999b). Microtubule motors in spindle and chromosome motility. Eur. J. Biochem. 262, 12-18.

Endow, S. A., Chandra, R., Komma, D. J., Yamamoto, A. H. and Salmon, E. D. (1994). Mutants of the Drosophila ncd microtubule motor protein cause centrosomal and spindle pole defects in mitosis. J. Cell Sci. 107, 859867.

Flanagan, C. A. and Ma, H. (1994). Spatially and temporally regulated expression of the MADS-box gene AGL2 in wild-type and mutant Arabidopsis flowers. Plant Mol. Biol. 26, 581-595.

Goodson, H. V., Kang, S. J. and Endow, S. A. (1994). Molecular phylogeny of the kinesin family of microtubule motor proteins. J. Cell Sci. 107, 18751884.

Grossniklaus, U., Vielle-Calzada, J. P., Hoeppner, M. A. and Gagliano, W. B. (1998). Maternal control of embryogenesis by MEDEA, a polycomb group gene in Arabidopsis. Science 280, 446-450.

Hagan, I. and Yanagida, M. (1992). Kinesin-related cut7 protein associates with mitotic and meiotic spindles in fission yeast. Nature 356, 74-76.

Hatsumi, M. and Endow, S. A. (1992). The Drosophila ncd microtubule motor protein is spindle-associated in meiotic and mitotic cells. J. Cell Sci. 103, 1013-1020.

Hildebrandt, E. R. and Hoyt, M. A. (2000). Mitotic motors in Saccharomyces cerevisiae. Biochim. Biophys. Acta 1496, 99-116.

Howard, J. (1996). The movement of kinesin along microtubules. Annu. Rev. Physiol. 58, 703-729.

Hoyt, M. A. and Geiser, J. R. (1996). Genetic analysis of the mitotic spindle. Annu. Rev. Genet. 30, 7-33.

Hoyt, M. A., He, L., Loo, K. K. and Saunders, W. S. (1992). Two Saccharomyces cerevisiae kinesin-related gene products required for mitotic spindle assembly. J. Cell Biol. 118, 109-120.
Karabay, A. and Walker, R. A. (1999a). Identification of microtubule binding sites in the Ncd tail domain. Biochemistry 38, 1838-1849.

Karabay, A. and Walker, R. A. (1999b). The Ncd tail domain promotes microtubule assembly and stability. Biochem. Biophys. Res. Commun. 258, 39-43.

Lee, Y. R. and Liu, B. (2000). Identification of a phragmoplast-associated kinesin-related protein in higher plants. Curr. Biol. 10, 797-800.

Liu, B., Cyr, R. J. and Palevitz, B. A. (1996). A kinesin-like protein, KatAp, in the cells of Arabidopsis and other plants. Plant Cell 8, 119-132.

Liu, B. and Lee, Y. R. J. (2001). kinesin-related protein in plant cytokinesis. J. Plant Growth Regul. 20, 141-150.

Liu, Y., Mitsukawa, N., Oosumi, T. and Whitier, R. F. (1995). Efficient isolation and mapping of Arabidopsis thaliana T-DNA insert junctions by thermal asymmetric interlaced PCR. Plant J. 8, 457-463.

Mandelkow, E. and Hoenger, A. (1999). Structures of kinesin and kinesinmicrotubule interactions. Curr. Opin. Cell Biol. 11, 34-44.

Marcus, A. I., Ambrose, J. C., Blickley, L., Hancock, W. and Cyr, R. J. (2002). The Arabidopsis thaliana protein, ATK1, is a minus-end directed kinesin that exhibits non-processive movement. Cell Motil. Cytoskeleton (in press).

Matthies, H. J., McDonald, H. B., Goldstein, L. S. and Theurkauf, W. E. (1996). Anastral meiotic spindle morphogenesis: role of the non-claret disjunctional kinesin-like protein. J. Cell Biol. 134, 455-464.

McCaffrey, G. and Vale, R. D. (1989). Identification of a kinesin-like microtubule-based motor protein in Dictyostelium-Discoideum. EMBO J. 8, 3229-3234.

Milisav, I. (1998). Dynein and dynein-related genes. Cell Motil. Cytoskeleton 39, 261-272.

Mitsui, H., Nakatani, K., Yamaguchi-Shinozaki, K., Shinozaki, K., Nishikawa, K. and Takahashi, H. (1994). Sequencing and characterization of the kinesin-related genes katB and katC of Arabidopsis thaliana. Plant Mol. Biol. 25, 865-876.

Mitsui, H., Yamaguchi-Shinozaki, K., Shinozaki, K., Nishikawa, K. and Takahashi, H. (1993). Identification of a gene family (kat) encoding kinesin-like proteins in Arabidopsis thaliana and the characterization of secondary structure of KatA. Mol. Gen. Genet. 238, 362-368.

Mountain, V. and Compton, D. A. (2000). Dissecting the role of molecular motors in the mitotic spindle. Anat. Rec. 261, 14-24.

O'Conell, M. J., Meluh, P. B., Rose, M. D. and Morris, N. R. (1993). Supression of the bimC4 mitotic spindle defect by deletion of $k l p A$, a gene encoding a KAR3-related kinesin-like protein in Aspergillus nidulans. J. Cell Biol. 120, 153-162.

Oppenheimer, D. G., Pollock, M. A., Vacik, J., Szymanski, D. B., Ericson, B., Feldmann, K. and Marks, M. D. (1997). Essential role of a kinesinlike protein in Arabidopsis trichome morphogenesis. Proc. Natl. Acad. Sci. USA 94, 6261-6266.

Peirson, B. N., Owen, H. A., Feldmann, K. A. and Makaroff, C. A. (1996). Characterization of three male-sterile mutants of Arabidopsis thaliana exhibiting alterations in meiosis. Sex. Plant Reprod. 9, 1-16.

Prigozhina, N. L., Walker, R. A., Oakley, C. E. and Oakley, B. R. (2001). $\gamma$-Tubulin and the C-terminal motor domain kinesin-like protein, KLPA, function in the establishment of spindle bipolarity in Aspergillus nidulans. Mol. Biol. Cell 12, 3161-3174.

Reddy, A. S. and Day, I. S. (2001). Kinesins in the Arabidopsis genome: a comparative analysis among eukaryotes. BMC Genomics $\mathbf{2}, 2$.

Reddy, A. S. N., Safadi, F., Narasimhulu, S. B., Golovkin, M. and Hu, X. (1996). A novel plant calmodulin-binding protein with a kinesin heavy chain motor domain. J. Biol. Chem. 271, 7052-7060.

Roof, D. M., Meluh, P. B. and Rose, M. D. (1991). Multiple kinesin-related proteins in yeast mitosis. Cold Spring Harbor Symp. Quant. Biol. 56, 693703.

Ross, K. J., Fransz, P. and Jones, G. H. (1996). A light microscopic atlas of meiosis in Arabidopsis thaliana. Chromosome Res. 4, 507-516.

Saunders, W. S. and Hoyt, M. A. (1992). Kinesin-related proteins required for structural integrity of the mitotic spindle. Cell 70, 451-458.

Saxton, W. M., Porter, M. E., Cohn, S. A., Scholey, J. M., Raff, E. C. and McIntosh, J. R. (1988). Drosophila kinesin - characterization of microtubule motility and ATPase. Proc. Natl. Acad. Sci. USA 85, 1109-1113.

Schachtman, D. P., Schroeder, J. I., Lucas, W. J., Anderson, J. A. and Gaber, R. F. (1994). Expression of an inward-rectifying potassium channel by the Arabidopsis KAT1 cDNA. Science 258, 1654-1658.

Scholey, J. M., Heuser, J., Yang, J. T. and Goldstein, L. S. B. (1989). Identification of globular mechanochemical heads of kinesin. Nature 338, 355-357. 
Scholey, J. M., Porter, M. E., Grissom, P. M. and McIntosh, J. R. (1985). Identification of kinesin in sea urchin eggs and evidence for its localization in the mitotic spindle. Nature 318, 483-486.

Sharp, D. J., Rogers, G. C. and Scholey, J. M. (2000). Microtubule motors in mitosis. Nature 407, 41-47.

Sharp, D. J., Yu, K. R., Sisson, J. C., Sullivan, W. and Scholey, J. M. (1999). Antagonistic microtubule-sliding motors position mitotic centrosomes in Drosophila early embryos. Nat. Cell Biol. 1, 51-54.

Song, H., Golovkin, M., Reddy, A. S. and Endow, S. A. (1997). In vitro motility of AtKCBP, a calmodulin-binding kinesin protein of Arabidopsis. Proc. Natl. Acad. Sci. USA 94, 322-327.

Sundaresan, V., Springer, P., Volpe, T., Haward, S., Jones, J., Dean, C., Ma, H. and Martienssen, R. (1995). Patterns of gene action in plant development revealed by enhancer trap and gene trap transposable elements. Genes Dev. 9, 1797-1810.
Tamura, K., Nakatani, K., Mitsui, H., Ohashi, Y. and Takahashi, H. (1999). Characterization of katD, a kinesin-like protein gene specifically expressed in floral tissues of Arabidopsis thaliana. Gene 230, 23-32.

Theurkauf, W. E. and Hawley, R. S. (1992). Meiotic spindle assembly in Drosophila females: behavior of nonexchange chromosomes and the effects of mutations in the nod kinesin-like protein. J. Cell Biol. 116, 1167-1180.

Vale, R., Reese, T. and Sheetz, M. (1985). Identification of a novel forcegenerating protein, Kinesin, involved in microtubule-based motility. Cell $\mathbf{4 2}$, 39-50.

Yang, J. T., Laymon, R. A. and Goldstein, L. S. B. (1989). A three-domain structure of kinesin heavy-chain revealed by DNA sequence and microtubule binding analyses. Cell 56, 879-889.

Yang, J. T., Saxton, W. M., Stewart, R. J., Raff, E. C. and Goldstein, L. S. B. (1990). Evidence that the head of kinesin is sufficient for force generation and motility in vitro. Science 249, 42-47. 